\title{
Extremely high Faraday and Kerr rotations and extraordinary optical transmission in four- layer dielectric-metal strips-dielectric-Bi:YIG planar structure
}

\author{
Victor Dmitriev, C. M. Santos, C. Nascimento \\ Department of Electrical Engineering, Federal University of Para, Av. Augusto Correa 01, Belem, 66075-090, \\ Brazil, e-mail: carlos.santos@icen.ufpa.br
}

\begin{abstract}
Faraday and Kerr effects as well as the extraordinary optical transmission in a structure that combines magneto-optical and plasmonic properties are studied. The planar structure consists of four layers, namely, dielectric layer, metal strips, once again dieletric layer and magneto-optic one. The magneto-optical layer is magnetized by a DC magnetic field normal to it. Our numerical analysis shows that the Faraday and Kerr rotations in this structure can be increased by more than two times in comparison with the published results. This is achieved due to high Q-factor resonances in magneto-optic layer.
\end{abstract}

Index Terms - Extraordinary optical transmission, Faraday effect, Kerr effect.

\section{INTRODUCTION}

The EOT (extraordinary optical transmission) effect is discussed in many published works (see, for example, a review paper [1]). In this effect, two principal geometries are considered, namely, 1D (one-dimensional) structure based on metal strips and 2D (two-dimensional) one formed by hole array in a metal. In the last decade, there was a considerable interest in the enhanced FR and KR (Faraday and Kerr rotation, respectively) combined with EOT in 1D and 2D metal structures placed on a MO (magneto-optical) layer. The physics behind this phenomenon is rather well understood [2], [3] and the experiments [4], [5] confirm the theory. In a simplified manner, the enhancement of FR and KR in these structures in comparison with a single MO layer magnetized by a DC magnetic field $\mathbf{H}_{\mathbf{0}}$ is explained as follows. The $\mathrm{E}_{\mathrm{y}}^{\mathrm{inc}}$ electric field component of the incident TEM wave produces on both sides of metal elements SPP (surface plasmon-polariton) mode with the current $\mathrm{j}_{\mathrm{y}}$ SPP (see Fig.1a). Due to overlap of the $\mathrm{E}_{\mathrm{y}}$ fields, this mode excites a waveguide TM mode with the wave vector $\mathrm{k}_{\mathrm{y}}{ }^{\mathrm{TM}}$ and with the components $\mathrm{E}_{\mathrm{y}}, \mathrm{H}_{\mathrm{x}}$ and $\mathrm{E}_{\mathrm{z}}$, (see Fig.1b) in MO layer (in fact it is a hybrid plasmon polariton mode [5]). This wave is partially excited also by the fields penetrating through slits in the metal. For simplicity, we show in Fig. 1 only the wave with the wave vector $+\mathrm{k}_{\mathrm{y}}{ }^{\mathrm{TM}}$, though $-\mathrm{k}_{\mathrm{y}}{ }^{\mathrm{TE}}$ is also excited resulting in resonant effects in bismuth iron garnet (BIG) layer. Due to tensor properties of the dielectric permittivity of MO material, i.e. due to non-diagonal elements of the tensor, a TE wave with 
the wave vector $\mathrm{k}_{\mathrm{y}}{ }^{\mathrm{TE}}$ and the components $\mathrm{E}_{\mathrm{x}}, \mathrm{H}_{\mathrm{y}}$ and $\mathrm{H}_{\mathrm{z}}$ is also excited in MO layer (Fig.1c). As a result, the transmitted (reflected) wave through (from) the structure acquires the component $\mathrm{E}_{\mathrm{x}}$ of electric field corresponding to FR (KR). The resonance nature of the effects provides a significant enhancement of FR and KR. One of the problem in these structures is to achieve frequency coincidence of the peaks of maximum reflectance and maximum of KR or maximum of EOT and maximum of FR. For this aim, it was suggested in [6] to fill in the air space in metal holes by a dielectric.

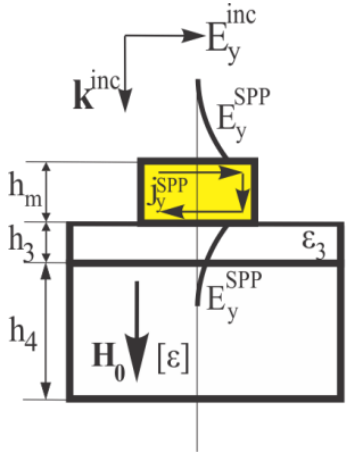

(a)

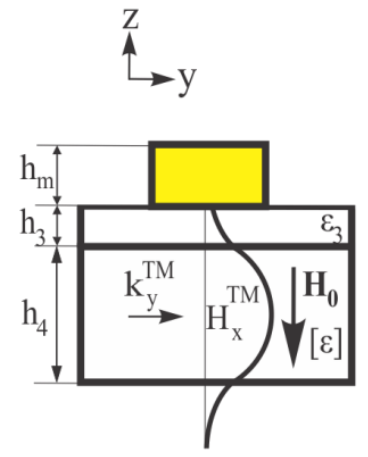

(b)

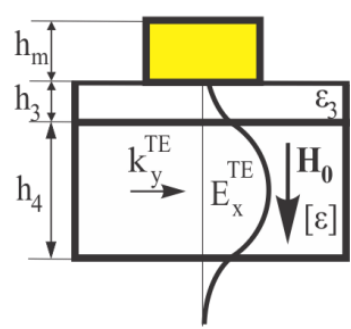

(c)

Fig. 1. Simplified schematic representation of fields and currents in two-layer structure with additional dielectric layer $h_{3}$ :

(a) excitation of SPP mode in metal by TEM incident wave with electric field $\mathrm{E}_{\mathrm{y}}$, (b) excitation of TM mode in MO layer by SPP mode in metal, (c) excitation of TE mode in MO layer by TM mode due to tensor $[\varepsilon]$.

FR for two-layer structures consisting of a metallic array and MO layer in the published literature is of the order of $\left(1^{\circ}\right.$ to $\left.2^{\circ}\right)$ and the transmittance at the level of (0.3 to 0.6$)$. One solution to enhance further FR is to add an additional dielectric layer between the metal elements and MO layer. In 2D structure, this allows one to increase FR rotation to $2.58^{\circ}$ with the transmittance of 0.46 at $\lambda=1099.6$ $\mathrm{nm}$ [7]. Notice that in a 1D structure, a record value of $4.2^{\circ}$ with transmittance of 0.3 for FR at $662 \mathrm{~nm}$ was reported and confirmed experimentally for low temperature $(30 \mathrm{~K})$ regime [5]. In the infra-red region the best results in the literature for the FR and transmittance is presented in [3] where for a $\lambda=$ $869 \mathrm{~nm}$ the FR is $3^{\circ}$ and transmittance is 0.6 .

Polar Kerr effect in two layers structures has been analyzed in sparse works [6], [8], [9]. In a 1D structure at $\lambda=880 \mathrm{~nm}$, the angle of $\mathrm{KR}$ is equal to $1.1^{\circ}$ and reflectance is smaller than 0.01 [8]. In [6] the authors investigated numerically the system where the metal has periodic square holes. In this case, the higher value of KR equal to $3.7^{\circ}$ was obtained, but the reflectance was also lower that 0.01 . On the other hand, in [9] in the structure made of a metal layer with circular holes, a relatively high reflectance 0.2 at $\lambda=917 \mathrm{~nm}$ but with a small $\mathrm{KR}$ of $-0.88^{\circ}$ was reached. Like the FR, the KR can also be enhanced by inserting a dielectric between the periodically perforated metal and MO layer. In three-layer structure the authors of [7] reached the record value of KR equal to $7.5^{\circ}$ at $\lambda=1099.6 \mathrm{~nm}$.

In this work, we numerically investigate the FR, KR and EOT in a planar structure composed of Brazilian Microwave and Optoelectronics Society-SBMO 
four layers (in the following we call it $4 \mathrm{~L}$ structure). Our optimized $4 \mathrm{~L}$ structure demonstrates FR more than two times higher as compared to that published in [3] and the KR is enhanced in more than five times. We explain the physical mechanism responsible for the improvement of the FR (KR).

\section{PROBLEM DESCRIPTION}

We propose insertion of an additional dielectric layer (see Fig.1) with the thickness $\mathrm{h}_{3}$ and permittivity $\varepsilon_{3}$ between the metal strips and MO layer (notice that the base structure discussed in [3] and which is used here for comparison, has $h_{3}=0$ ). Another important element which we introduce in the structure is one more dielectric layer denoted as layer 1 with the thickness $\mathrm{h}_{1}$ (Fig.2). With these dielectric layers, we have several additional parameters at hand to optimize the structure. In summary, our proposed structure consists of four layers. A periodic metal grating is placed on dielectric 3 with permittivity constant $\varepsilon_{3}$. A dielectric with permittivity $\varepsilon_{2}$ fills in the slits between the metal strips of the grid. This three-layer set is placed on a thin layer of Bi-substituted yttrium iron garnet (Bi:YIG) magnetized by the magnetic field $\mathbf{H}_{\mathbf{0}}$ in $-\mathrm{z}$ direction. Layer 3 has the dielectric constant much lower than that of the Bi:YIG and this leads to a reduction of coupling between the metal grating and the MO waveguide.

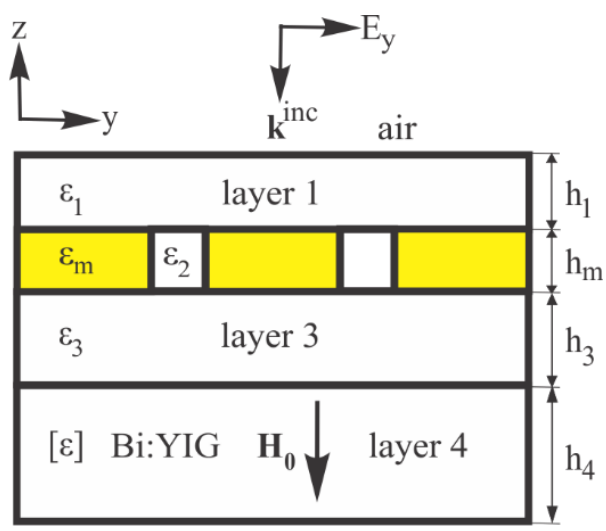

(a) air

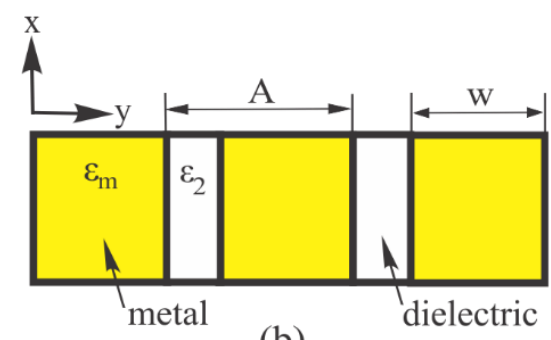

(b)

Fig. 2. Four-layer structure, a) side view, b) top view without layer 1.

In the numerical analysis, optical properties of the metal, which is gold ( $\mathrm{Au})$, are modelled by the following dielectric function $\varepsilon_{m}$ [2], [7], [8], [9]:

$$
\varepsilon_{m}=\varepsilon_{\infty}-\frac{\omega_{p}^{2}}{\left(\omega^{2}+i \gamma \omega\right)},
$$


where $\varepsilon_{\infty}$ the high-frequency limit dielectric, $\omega_{p}$ the plasma frequency, $\omega$ frequency of incident wave and $\gamma$ the damping term. For the film of gold in the wavelength region of interest, the parameters of Eq. (1) have the following values: $\varepsilon_{\infty}=7.9, \omega_{p}=1.3324 .10^{16} \mathrm{~s}^{-1}$ and $\gamma=1.1395 .10^{14} \mathrm{~s}^{-1}$. These parameters correspond to the experimental data of [10].

The MO material is described by the permittivity tensor

$$
[\varepsilon]=\left[\begin{array}{ccc}
\varepsilon_{r} & i g & 0 \\
-i g & \varepsilon_{r} & 0 \\
0 & 0 & \varepsilon_{r}
\end{array}\right],
$$

where $\varepsilon_{r}=5.5+\mathrm{i} 0.0025$ and $g=0.01-\mathrm{i} 0.0015$ [2], [7], [8], [9]. The magnetic permeability of the Bi:YIG layer, the gold and the dielectrics are considered to be equal to 1 .

Several dielectrics used in optics possess the permittivity in the region of 1.9 to 5 (for example, $\mathrm{MgF}_{2}, \mathrm{SiO}, \mathrm{SiO}_{2}, \mathrm{TiO}_{2}, \mathrm{Al}_{2} \mathrm{O}_{3}$, etc). Thus, in the optimization process we varied the permittivity constants in this interval. The dimensions and parameters of the $4 \mathrm{~L}$ structure are given in Table I.

TABLE I. PARAMETERS OF BASE STRUCTURE AND 4L STRUCTURE (DIMENSIONS ARE IN NANOMETERS).

\begin{tabular}{|l|c|c|c|c|c|c|c|c|c|}
\hline & $\mathrm{A}$ & $\mathrm{W}$ & $\mathrm{h}_{1}$ & $\mathrm{~h}_{\mathrm{m}}$ & $\mathrm{h}_{3}$ & $\mathrm{~h}_{4}$ & $\varepsilon_{1}$ & $\varepsilon_{2}$ & $\varepsilon_{3}$ \\
\hline base structure & 750 & 600 & - & 75 & - & 515 & - & - & - \\
\hline 4L structure & 735 & 620 & 150 & 82 & 111 & 545 & 3 & 4.9 & 1.9 \\
\hline
\end{tabular}

The structure is illuminated by a plane wave with normal incidence polarized in y-direction, i.e. perpendicular to the slits of metal. The numerical analyses was fulfilled using the commercial software COMSOL [11].

\section{RESULTS AND DISCUSSION}

In this section, we investigate the influence of gap $\mathrm{h}_{3}$ on FR (KR). The gap between the metallic strip and MO layer reduces the coupling of the SPP mode of the metal and TM mode of the MO waveguide. Consequently, the Q-factor of the resonance related to the Faraday or Kerr effect generated in the structure can be enhanced. In the base structure where the metal strips are placed directly on the MO sheet, these elements are strongly coupled. Thus, the coupling constant has its 
maximum value. As a result, the quality factor of the TM mode resonance is relatively low and the resonance curve corresponding to FR has a low maximum. The introduction of the gap reduces the coupling constant and, as a consequence, increases the quality factor of resonance. The higher quality factor leads to to a higher value of the FR and KR resonance peaks.

In our discussion firstly we shall consider the gap $h_{3}$ filled by air and the parameters of the base structure used in [8] are given in Table 1. Fig.3 demonstrates the $h_{3}$ dependence of FR for this modified base structure. One can see that when $h_{3}$ is increased, the quality factor of the resonance and the angle of FR increase until a certain extreme value which, is one order higher than that for the structure withour air gap. With increasing $h_{3}$ the positive resonance is gradually shifted to higher wavelengths whereas the negative resonance is shifted to lower wavelengths.

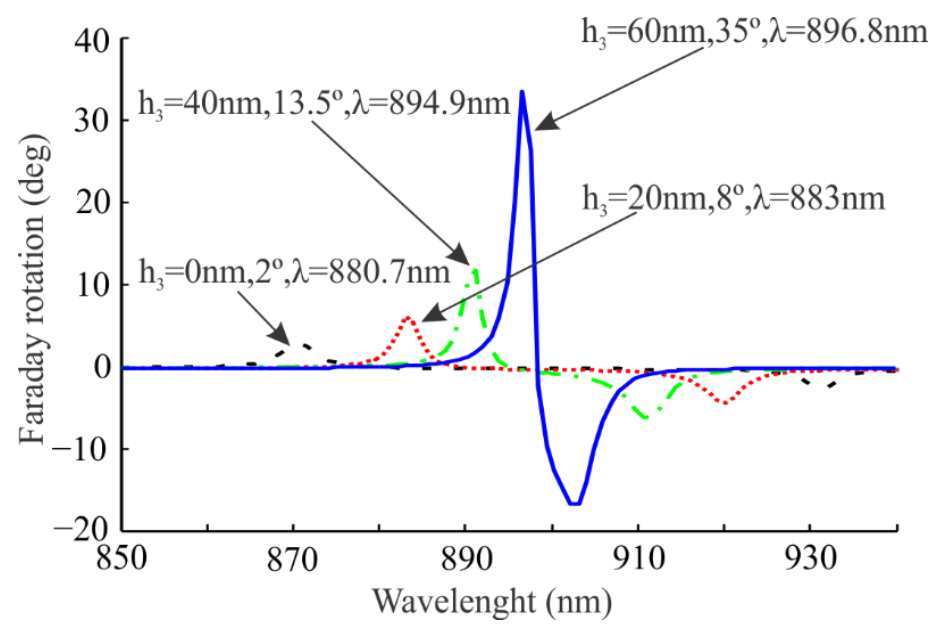

Fig. 3. Faraday rotation spectra in base structure for different gaps $h_{3}$.

The role of dielectric layer 1 is to improve the figure of merit (FOM) due to better impedance matching of the structure with the incident wave. The FOM is defined for the transmitted wave as $\left|\theta_{F}\right| \sqrt{T}$, where $\theta_{F}$ is the angle of FR and $T$ is the transmittance [3], [12]. Dependence of the FOM with respect $\mathrm{h}_{1}$ is shown in Fig.4. One can see that there is an optimum value of $\mathrm{h}_{1}$ corresponding to maximum of the FOM. 


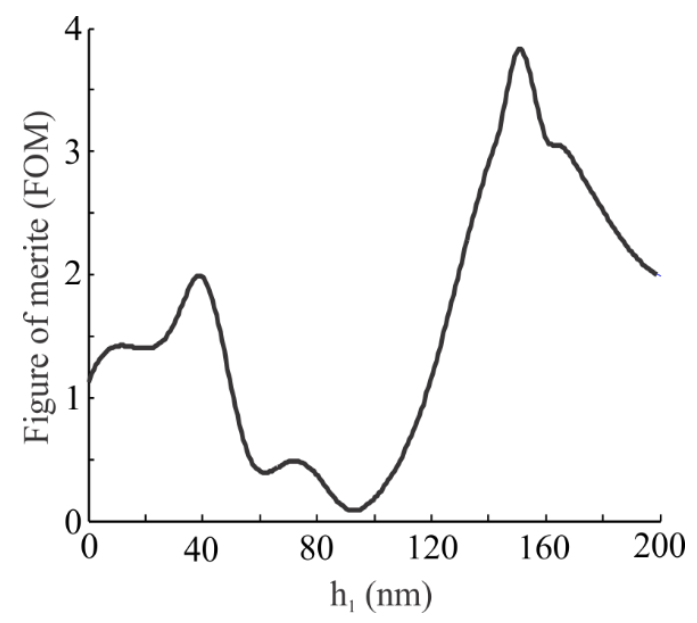

Fig. 4. Figure of merit for $4 \mathrm{~L}$ structure versus of $h_{1}, \varepsilon_{1}=3$. For every value of $h_{1}$ there is a wavelength to which the figure of merit has maximum value. Thus, for example, for $h_{1}=150 \mathrm{~nm}$ (optimal value) the FOM has your maximum value for wavelength equal to $945 \mathrm{~nm}$.

The field distributions for the base structure and for $4 \mathrm{~L}$ configuration with the parameters given in Table 1 are shown in Fig.5a, 5b, 5c and 5d. In this case, the field distribution inside the structure 4L is defined by the values of the geometric parameters (for example, pitch and thicknesses) as well as the values of permittivity of the dielectrics present in the structure. Comparing Fig.5c and Fig.5d one can see that the maximum intensity of the field $\mathrm{E}_{\mathrm{x}}$ in the suggested structure is six times higher than in base structure.

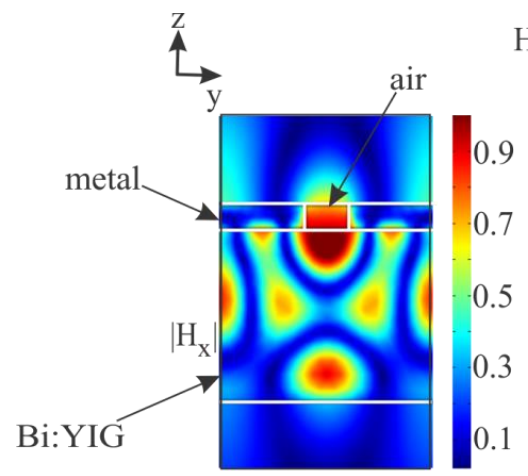

(a)

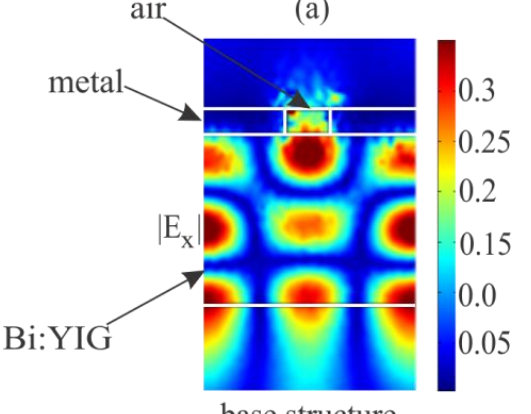

base structure

(c)

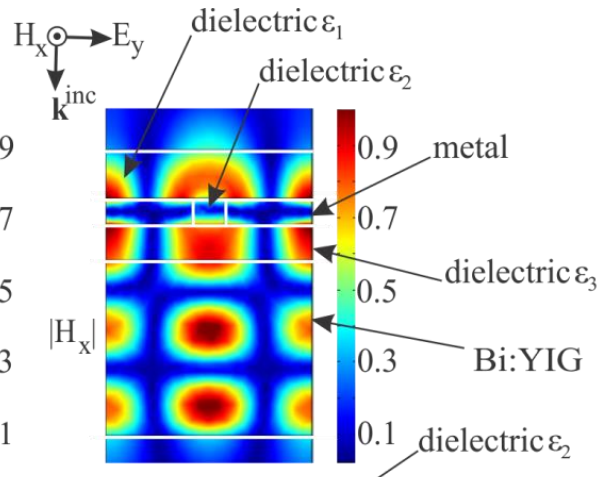

(b)

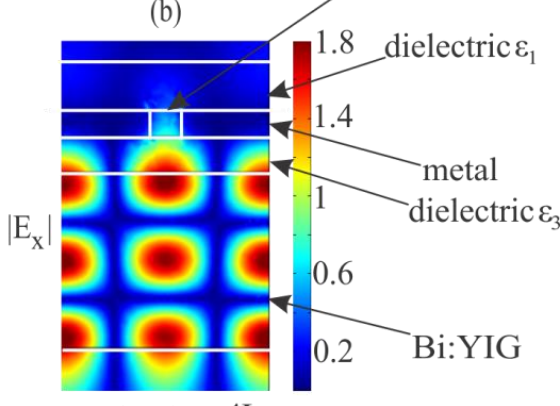

structure $4 \mathrm{~L}$

(d) 
Fig. 5. Spatial distributions of $\left|H_{x}\right|$ in (a) and $\left|E_{x}\right|$ in (c) for base structure at $\lambda=870.7 \mathrm{~nm}$. Spatial distributions of $\left|H_{x}\right|$ in (b) and $\left|E_{x}\right|$ in (d) for structure $4 \mathrm{~L}$ at $\lambda=945 \mathrm{~nm}$. Amplitudes of fields are normalized to that of incident light.

The FR and transmittance spectrum for the base structure and 4L are given in Fig.6. The base structure possesses the FR equal to $2.75^{\circ}$ and transmittance 0.57 at $\lambda=870.7 \mathrm{~nm}$ (see Fig.6a). Note that the values of the FR and transmittance calculated for base structure using COMSOL are consistent with those obtained in [8] where the authors used another numerical method. On the other hand, the $4 \mathrm{~L}$ structure in $\lambda=945 \mathrm{~nm}$ has FR equal to $7.8^{\circ}$ and the transmittance equal to 0.25 , Fig.6b. These results demonstrate that in $4 \mathrm{~L}$ structure the FR is increased more than two times in comparison to the base structure.

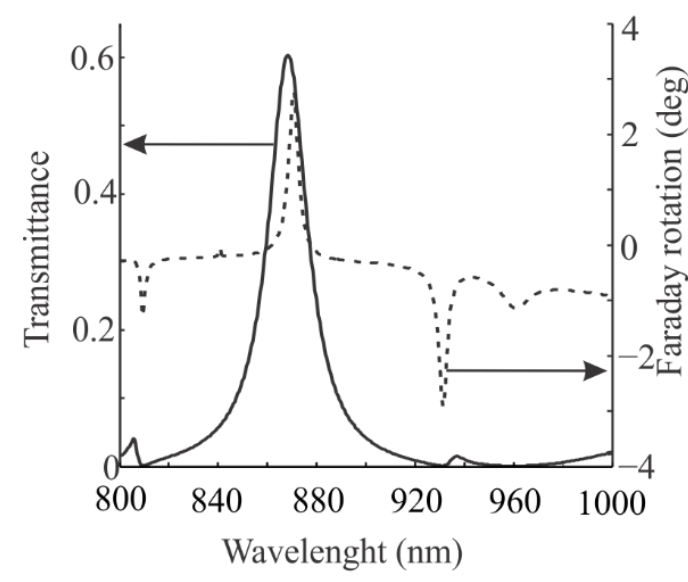

(a)

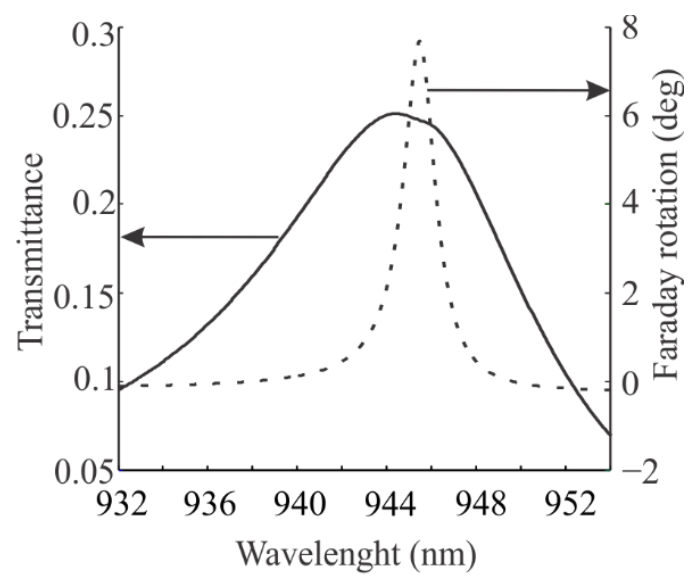

(b)

Fig. 6. Transmittance and Faraday rotation spectra, (a) base structure, (b) 4L structure.

For these two configurations the FR is accompanied by EOT, however level of transmittance for the case analyzed in [8] is better than that for our $4 \mathrm{~L}$ structure. But, comparing the two configurations from the point view of FOM, the $4 \mathrm{~L}$ structure has $\mathrm{FOM}=3.9$ whereas the base structure has $\mathrm{FOM}=$ 2.05. Thus, the proposed structure has the FOM increased almost 2 times compared to the configuration presented in [8].

In Fig.7a we show the spectra of KR and reflectance of the base structure. At $\lambda=870.9 \mathrm{~nm}$, it has $\mathrm{KR}$ equals to $-3.2^{\circ}$ and the reflectance 0.03 . In $4 \mathrm{~L}$ configuration we succeded not only the FR improvement but also a better value of the KR. The spectra of KR and reflectance for $4 \mathrm{~L}$ are given in Fig. 7 b. For $\lambda=945 \mathrm{~nm}$ the KR equals to $-23.8^{\circ}$ and the reflectance is 0.04 . Evidently, the proposed structure demonstrates much better KR and the reflectance in comparison with the results of [8]. The 
value $-23.8^{\circ}$ of $\mathrm{KR}$ is a record for the known structures with metallic periodic elements and $\mathrm{MO}$ material.

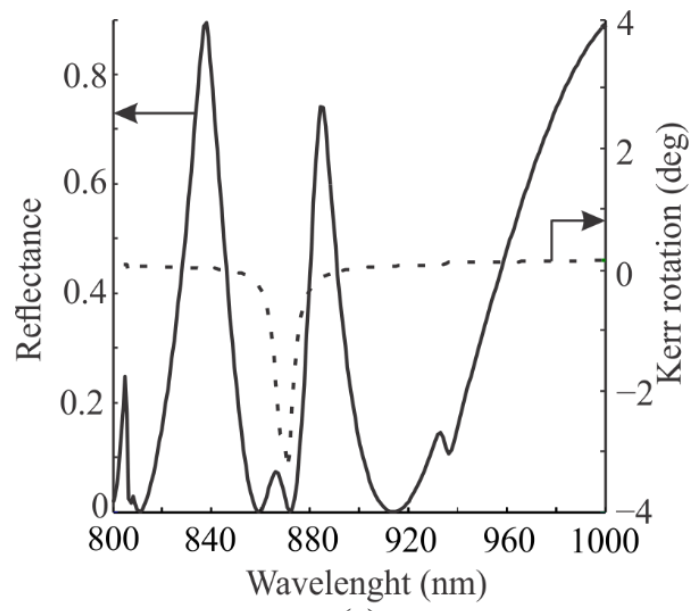

(a)

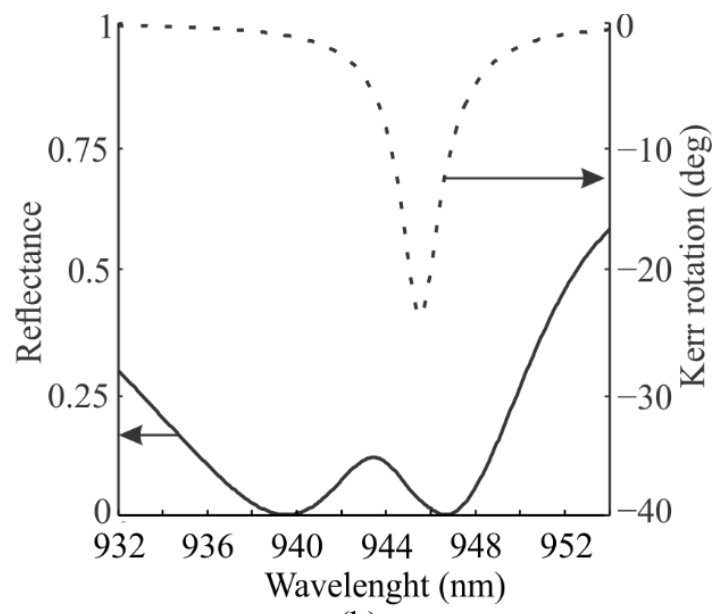

(b)

Fig. 7. Spectra of reflectance and KR rotation, (a) base structure and (b) 4L structure.

In addition, we calculated the FOM to the wavelength with higher KR. In the case of wave reflection, the FOM can be described in terms of the Kerr rotation $\theta_{K}$ and reflectance R, i.e $\left|\theta_{K}\right| \sqrt{R}$. For the $4 \mathrm{~L}$ structure, the FOM is 8.6 times higher than that for base structure.

\section{CONCLUSION}

We have shown theoretically that it is possible to reach simultaneously high value Faraday rotation with EOT simultaneously in the new four-layer planar structure. It exhibits extremely high Kerr rotation as compared with the other reported structures. The increase of the Faraday (Kerr) rotation is due to the presence of the dielectric between the metal strips and MO material that reduces the coupling constant of the system and consequently increases the Q-factor of the resonance. We believe that the suggested structure can find application in magnetic field and current sensors, in magnetic microscopy, optical modulators, data storage device and etc. Enhanced resonant electromagnetic field in the MO layer can promote nonlinear MO effects.

\section{ACKNOWLEDGMENT}

This work was supported by Brazilian agencies CAPES and CNPq.

\section{REFERENCES}


[1] F.J. Garcia-Vidal, L. Martin-Moreno, T.W. Ebbesen, and L. Kuipers, Light passing through subwavelength apertures, Rev. Mod. Phys., 82 (2010), 729-787.

[2] V. L. Belotelov, L.L. Doskolovich, and A.K. Zvezdin, Extraordinary magneto-optical effects and transmission through metal-dielectric plasmonic systems, Phys. Rev. Lett., vol. 98, (2007), pp. 1-4.

[3] H. Xu and B.S. Ham, Investigation of extraordinary optical transmission and Faraday effect in one-dimensional metallicmagnetic gratings, Opt. express, vol. 16, (2008), pp. 21375-21382.

[4] J. Y. Chin, T. Steinle, T. Wehlus, D. Dregely, T. Weiss, V. I. Belotelov, B. Stritzker and H. Giessen, Nonreciprocal plasmonics enables giant enhancement of thin-film Faraday rotation, Nat. Commun., vol. 4, (2013), pp. 1-6.

[5] D. Floess, J. Y. Chin, A. Kawatani, D. Dregely, H. Habermeier, T. Weiss, and H. Giessen, Tunable and switchable polarization rotation with non-reciprocal plasmonic thin films at designated wavelengths, Light Sci Appl, vol. 4, (2015), pp. $1-7$.

[6] D. Li, C. Lei, L. Chen, Z. Tang, S. Zhang, S. Tang, and Y. Du, Waveguide plasmon resonance induced enhancement of the magneto-optics in a Ag/Bi: YIG bilayer structure, JOSA B, vol. 32, (2015), pp. 2003-2008.

[7] V. Dmitriev, F. Paixão, and M. Kawakatsu, Enhancement of Faraday and Kerr rotations in three-layer heterostructure with extraordinary optical transmission effec, Opt. Lett., vol. 38, (2013), pp. 1052-1054.

[8] V. L. Belotelov, L.L. Doskolovich, V.A Kotov, E.A. Bezus, D.A. Bykov, and A.K Zvezdin, Faraday rotation effect enhancement in metal-dielectric plasmonic systems, Proc. Of SPIE, vol. 6581, (2007), pp. 1-8.

[9] D. Li, L. Chen, C. Lei, J.L. Menendez, C. Mallada, Z. Tang, S. Tang, and Y. Du. Plasmon-enhanced magneto-optical activity in nanostructure with circle annular arrays, JOSA B, vol. 33, (2016), pp. 922-927.

[10] D. Palik, Handbook of optical constants of solids, Edward Academic Press Inc., Orlando, FL, 1985.

[11] https://www.comsol.com

[12] A. K. Zvezdin and and V. A. Kotov, Modern Magnetooptics and Magnetooptical Materials, Taylor \& Francis, New York, 1997. 